\title{
氧化镧掺杂铌酸钾钠陶瓷的电、光性能研究
}

\author{
肖舒琳 ${ }^{1}$ ，戴中华 ${ }^{1}$ ，李定妍 ${ }^{1}$, 张凡博 ${ }^{1}$, 杨利红 ${ }^{1}$ ，任晓兵 ${ }^{2}$
}

(1. 西安工业大学 光电工程学院, 陕西省薄膜技术与光学检测重点实验室, 西安 710021 ; 2. 西安交通大学前沿 技术研究院，西安 710049)

摘 要: 铌酸钾钠 $\left(\mathrm{K}_{0.5} \mathrm{Na}_{0.5} \mathrm{NbO}_{3}, \mathrm{KNN}\right)$ 基陶瓷具有充放电速度快、透明度高、应用温度范围宽、使用寿命长等优点, 在脉冲功率器件等领域具有广阔的应用前景。通过改性技术提高铌酸钾钠基陶瓷的电、光性能是该方向的研究热 点。本研究采用固相法制备 $0.825\left(\mathrm{~K}_{0.5} \mathrm{Na}_{0.5}\right) \mathrm{NbO}_{3}-0.175 \mathrm{Sr}_{1-3 x / 2} \mathrm{La}_{x}\left(\mathrm{Sc}_{0.5} \mathrm{Nb}_{0.5}\right) \mathrm{O}_{3}(x=0,0.1,0.2,0.3)$ 陶瓷(简称 $0.825 \mathrm{KNN}$ $0.175 \mathrm{SLSN}$ ), 研究 $\mathrm{La}_{2} \mathrm{O}_{3}$ 掺杂对其相结构、微观形貌、光学、介电、铁电及储能性能的影响。研究结果表明: $0.825 \mathrm{KNN}-$ $0.175 \mathrm{SLSN}$ 陶瓷具有高对称性的伪立方相结构; 随着 $\mathrm{La}_{2} \mathrm{O}_{3}$ 掺杂量增大, 陶瓷的平均晶粒尺寸减小, 相变温度 $\left(T_{\mathrm{m}}\right)$ 及饱和极化强度 $\left(P_{\max }\right)$ 增大, 达到峰值后下降。在 $x=0.3$ 时, 该体系陶瓷表现出优异的透明性, 在可见光波长 $(780 \mathrm{~nm})$ 及近红外波长 $(1200 \mathrm{~nm})$ 范围内透过率分别达 $65.2 \%$ 及 $71.5 \%$, 同时实现了 $310 \mathrm{kV} / \mathrm{cm}$ 的击穿场强和 $1.85 \mathrm{~J} / \mathrm{cm}^{3}$ 的可 释放能量密度。

关 键 词: 铌酸钾钠; 无铅透明陶瓷; 透过率; 储能性能

中图分类号: TQ174 文献标志码: A

\section{Electrical and Optical Property of Lanthanum Oxide Doped Potassium Sodium Niobate Ceramics}

\author{
XIAO Shulin ${ }^{1}$, DAI Zhonghua ${ }^{1}$, LI Dingyan ${ }^{1}$, ZHANG Fanbo ${ }^{1}$, YANG Lihong ${ }^{1}$, REN Xiaobing ${ }^{2}$
}

(1. Shaanxi Province Key Laboratory of Thin Films Technology \& Optical Test, School of Optoelectronic Engineering, Xi'an Technological University, Xi'an 710021, China; 2. Frontier Institute of Science and Technology, Xi'an Jiaotong University, X'ian 710049, China)

\begin{abstract}
Potassium sodium niobate $\left(\mathrm{K}_{0.5} \mathrm{Na}_{0.5} \mathrm{NbO}_{3}, \mathrm{KNN}\right)$ based ceramics can be widely used for pulsed power systems due to their fast charge-discharge rate, high transparency, wide range of working temperature, and long cycle life. Improving the electrical and optical property of KNN-based ceramics through modification is a research hotspot in this field. $0.825\left(\mathrm{~K}_{0.5} \mathrm{Na}_{0.5}\right) \mathrm{NbO}_{3}-0.175 \mathrm{Sr}_{1-3 x / 2} \mathrm{La}_{x}\left(\mathrm{Sc}_{0.5} \mathrm{Nb}_{0.5}\right) \mathrm{O}_{3} \quad(x=0, \quad 0.1, \quad 0.2, \quad 0.3) \quad(0.825 \mathrm{KNN}-$ $0.175 \mathrm{SLSN}$ ) ceramics were synthesized by solid state method. The effect of $\mathrm{La}_{2} \mathrm{O}_{3}$ doping on the phase structure, microstructure, optical property, dielectric property, ferroelectric property and energy storage property of the ceramic was studied. The results indicated that the structure of $0.825 \mathrm{KNN}-0.175 \mathrm{SLSN}$ ceramics was pseudo-cubic phase with high symmetry. With increment of $\mathrm{La}_{2} \mathrm{O}_{3}$ content, the average grain size of $0.825 \mathrm{KNN}-0.175 \mathrm{SLSN}$ ceramics
\end{abstract}

收稿日期：2021-05-11; 收到修改稿日期：2021-07-09; 网络出版日期：2021-08-20

基金项目：国家自然科学基金(51831006，51431007); 陕西省科技计划(2020GY-311); 西安市智能兵器重点实验室基金 (2019220514SYS020CG042)

National Natural Science Foundation of China (51831006, 51431007); Science and Technology Project of Shaanxi Province (2020GY-311); Xi' an Key Laboratory of Intelligence (2019220514SYS020CG042)

作者简介: 肖舒琳(1998-), 女, 硕士研究生. E-mail: 1319643700@qq.com XIAO Shulin (1998-), female, Master candidate. E-mail: 1319643700@qq.com

通信作者：戴中华，教授. E-mail: zhdai@mail.xjtu.edu.cn DAI Zhonghua, professor. E-mail: zhdai@mail.xjtu.edu.cn 
decreased, and the phase transition temperature $\left(T_{\mathrm{m}}\right)$ and saturation polarization intensity $\left(P_{\max }\right)$ increased and then decreased. $0.825 \mathrm{KNN}-0.175 \mathrm{SLSN}$ ceramics exhibit excellent transparency at $x=0.3$, the transmittance in the visible wavelength $(780 \mathrm{~nm})$ and near-infrared wavelength $(1200 \mathrm{~nm})$ ranges reaches $65.2 \%$ and $71.5 \%$, respectively. The dielectric breakdown strength of $310 \mathrm{kV} / \mathrm{cm}$ and a recoverable energy density of $1.85 \mathrm{~J} / \mathrm{cm}^{3}$ are achieved at $x=0.3$.

Key words: potassium sodium niobate; lead-free transparent ceramics; transmittance; energy storage property

随着脉冲功率技术的快速发展和元件透明化需 求的增加，具有优异储能特性的无铅透明铁电陶 瓷作为脉冲功率系统的关键元件, 被广泛应用于 航天航空、定向武器和新能源汽车等领域 ${ }^{[1-4]}$ 。目 前常见的储能材料体系有 $\mathrm{NaNbO}_{3}(\mathrm{NN})$ 体系、 $\mathrm{K}_{0.5} \mathrm{Na}_{0.5} \mathrm{NbO}_{3}(\mathrm{KNN})$ 体系、 $\mathrm{BaTiO}_{3}(\mathrm{BT})$ 体系与 $\mathrm{Bi}_{0.5} \mathrm{Na}_{0.5} \mathrm{TiO}_{3}(\mathrm{BNT})$ 体系等。上述材料中, $\mathrm{KNN}$ 基陶 瓷材料具有较高的居里温度、稳定的高压电系数、 易固溶、缺陷容忍度高等优点，是易于实现光学、 电学以及机械性能耦合的多功能材料 ${ }^{[5-7]}$ 。纯 KNN 陶瓷的剩余极化强度 $P_{\mathrm{r}}$ 较大, 饱和极化强度与剩余 极化强度差 $\left(P_{\max }-P_{\mathrm{r}}\right)$ 小于 $5 \mu \mathrm{C} / \mathrm{cm}^{2}$, 且击穿场强 $E_{\mathrm{b}}<40 \mathrm{kV} / \mathrm{cm}$ ，导致其不能作为优良的储能介质材 料 ${ }^{[8]}$ 。此外, KNN 陶瓷在室温下为高对称性的正交 相结构, 粒径为 4 5 $\mu \mathrm{m}^{[9]}$, 难以采用普通的烧结方 法制成透明陶瓷 ${ }^{[10-11]}$ 。

为了改善 $\mathrm{KNN}$ 材料的电学及光学性能, 研究 者尝试通过稀土元素掺杂改性基体材料。 $\mathrm{Lu}$ 等 ${ }^{[12]}$ 通过在 $\mathrm{Bi}_{0.5} \mathrm{Na}_{0.5} \mathrm{TiO}_{3}-\mathrm{BaTiO}_{3}$ 材料中掺杂适量的 $\mathrm{La}$ 和 $\mathrm{Zr}$ 元素, 最终得到了可利用储能密度 $W_{\mathrm{rec}}=$ $1.21 \mathrm{~J} / \mathrm{cm}^{3}$ 。Wang 等 ${ }^{[13]}$ 对 BNT 基陶瓷改性, 设计了 $\left[\left(\mathrm{Bi}_{0.5} \mathrm{Na}_{0.5}\right)_{0.94} \mathrm{Ba}_{0.06}\right] \mathrm{La}_{(1-x)} \mathrm{Zr}_{x} \mathrm{TiO}_{3}$ 储能陶瓷, 其具 有高饱和极化强度 $\left(P_{\max }=37.5 \mu \mathrm{C} / \mathrm{cm}^{2}\right)$, 并表现出双电 滞回线形状，可利用储能密度 $W_{\mathrm{rec}}$ 提高至 $1.58 \mathrm{~J} / \mathrm{cm}^{3}$ 。 为实现多晶陶瓷材料的透明性, Ren 等 ${ }^{[14]}$ 通过在 $\mathrm{K}_{0.5} \mathrm{Na}_{0.5} \mathrm{NbO}_{3}$ 中引入 $\mathrm{SrZrO}_{3}$ 作为第二组元, 将晶粒 尺寸降至 $0.19 \mu \mathrm{m}$, 从而使 $(1-x) \mathrm{K}_{0.5} \mathrm{Na}_{0.5} \mathrm{NbO}_{3}-x \mathrm{SrZrO}_{3}$ 陶瓷获得较高的光学透明性 $(T \sim 68 \%)$ 。Zhang 等 ${ }^{[15]}$ 通过在 $\mathrm{KNN}$ 基陶瓷中固溶第二组元 $\left(\mathrm{K}_{0.7} \mathrm{Bi}_{0.3}\right) \mathrm{NbO}_{3}$, 使其在晶界处聚集来抑制晶粒生长, 利用固相反应 法制备的 $0.85 \mathrm{KNN}-0.15 \mathrm{KBN}$ 透明陶瓷在近红外光 波长范围内透光率达到 $83.3 \%$ 。Heartling 等 ${ }^{[16]}$ 通过 热压烧结技术, 在锆钛酸铅(PZT)陶瓷基体中掺入 La 元素, 制备出锆钛酸铅锞(PLZT)透明陶瓷, 大大 提高了铅基陶瓷的透明度。Song 等 ${ }^{[17]}$ 在 PMN-PT 驰豫铁电陶瓷中加入 $\mathrm{La}$ 元素后制备了高透明度的 陶瓷，当掺杂量为 $4 \%$, 厚度为 $0.5 \mathrm{~mm}$ 时, 陶瓷透光 率在可见光范围内接近 $70 \%$ 。
本研究通过稀土元素异价离子取代方法, 在 $0.825\left(\mathrm{~K}_{0.5} \mathrm{Na}_{0.5}\right) \mathrm{NbO}_{3}-0.175 \mathrm{Sr}\left(\mathrm{Sc}_{0.5} \mathrm{Nb}_{0.5}\right) \mathrm{O}_{3}$ 陶瓷中掺 入 $\mathrm{La}_{2} \mathrm{O}_{3}$ 。采用传统固相反应法制备 $0.825\left(\mathrm{~K}_{0.5} \mathrm{Na}_{0.5}\right) \mathrm{NbO}_{3}-0.175 \mathrm{Sr}_{1-3 x / 2} \mathrm{La}_{x}\left(\mathrm{Sc}_{0.5} \mathrm{Nb}_{0.5}\right) \mathrm{O}_{3}(x=$ $0,0.1,0.2,0.3)$ 陶瓷，简称 $0.825 \mathrm{KNN}-0.175 \mathrm{SLSN}$ 陶 瓷, 研究掺杂 $\mathrm{La}_{2} \mathrm{O}_{3}$ 含量对 $0.825 \mathrm{KNN}-0.175 \mathrm{SLSN}$ 陶瓷相结构、微观形貌、光学、介电、铁电及储能 性能的影响规律。

\section{1 实验方法}

\section{1 样品制备}

以分析纯 $\mathrm{K}_{2} \mathrm{CO}_{3}(99.5 \%) 、 \mathrm{Na}_{2} \mathrm{CO}_{3}(99.8 \%)$ 、 $\mathrm{Nb}_{2} \mathrm{O}_{5}(99.5 \%) 、 \mathrm{Sr}_{2} \mathrm{CO}_{3}(99 \%) 、 \mathrm{Sc}_{2} \mathrm{O}_{3}(99.9 \%)$ 和 $\mathrm{La}_{2} \mathrm{O}_{3}(99 \%)$ 为原料, 采用传统固态反应法制备 $0.825 \mathrm{KNN}-0.175 \mathrm{SLSN}$ 陶瓷 $(x=0,0.1,0.2,0.3)$ 。根据 化学计量比进行称料, 将混合粉料进行一次球磨, 以 $\phi 2 \sim 5 \mathrm{~mm}$ 的锆球为介质, 在酒精中球磨 $18 \mathrm{~h}$ 。将 料浆置于培养皿中, 在 $80{ }^{\circ} \mathrm{C}$ 烘干后进行篎料。将得 到的粉料置于密闭的氧化铝坩埚中在 $950{ }^{\circ} \mathrm{C}$ 保温 $5 \mathrm{~h}$ 进行预烧, 再进行 $10 \mathrm{~h}$ 二次球磨。干燥后, 将粉 料与质量百分比 $5 \%$ 的聚乙烯醇水溶液均匀混合。 为了使粘结剂充分扩散, 将混合后的粉料压制成 坏体后放置 $12 \mathrm{~h}$ 。混合粉料在 $250 \mathrm{MPa}$ 下压制成 $\phi 12 \mathrm{~mm} \times 1 \mathrm{~mm}$ 的圆柱生坏。压制的生坏样品在 $600{ }^{\circ} \mathrm{C}$ 下保温 $5 \mathrm{~h}$ 排胶后, 再在 1200 1300 ${ }^{\circ} \mathrm{C}$ 烧结 $5 \mathrm{~h}$ 。为了获得高的击穿场强, 本研究对烧结后的样 品进行打磨抛光处理, 使其表面平行光滑, 厚度约 为 $0.15 \mathrm{~mm}$ 。采用丝网印刷方法在样品表面涂覆银 浆, $800{ }^{\circ} \mathrm{C}$ 烧制 $20 \mathrm{~min}$ 后得到银电极。

\section{2 性能测试}

采用 Archimedes 排水法测试样品密度; 采用 X 射线衍射仪(XRD, D8 Advance, Bruker, Germany)和 扫描电子显微镜(SEM, Quanta 250F, FEI, USA)测试 烧结后样品的相结构及微观形貌; 采用 LCR 电桥 (E4980A, Aglient, USA)在-150 150 ${ }^{\circ} \mathrm{C}$ 温度范围以 及 1 1000 kHz 的频率下测试样品的介电常数; 采用 分光光度计(UV-2550, Tokyo, Japan)测试样品的透 
过率, 测试波长范围为 400 1200 $\mathrm{nm}$ 。采用铁电测 试仪(Premier II, Radiant, USA)测试样品室温下电滞 回线, 测试频率为 $5 \mathrm{~Hz}$ 。

\section{2 结果与讨论}

\section{$2.10 .825 K N N-0.175 S L S N$ 陶瓷的结构分析}

材料的致密度越高, 材料内部的气孔杂质越少, 可以降低材料气孔对于光线的吸收, 从而提高光线 透过率 ${ }^{[18-20]}$; 还可有利于提高的击穿场强, 从而获 得较大的储能密度。图 1 为 $0.825 \mathrm{KNN}-0.175 \mathrm{SLSN}$ 陶瓷样品在室温下测得的密度-掺杂量关系曲线。由 图可知随着 $\mathrm{La}_{2} \mathrm{O}_{3}$ 含量增大, $0.825 \mathrm{KNN}-0.175 \mathrm{SLSN}$ 陶 瓷的密度先降低后逐渐提高, 在 $x=0.3$ 处陶瓷密度 最大。

图 2 为 $0.825 \mathrm{KNN}-0.175 \mathrm{SLSN}$ 陶瓷的 XRD 图谱, 可以发现 XRD 衍射谱无杂峰, 均呈单一的钙铁矿 结构。由此可见, 掺入 $\mathrm{La}_{2} \mathrm{O}_{3}$ 均形成了单一结构固溶 体。所有样品在 $2 \theta=45^{\circ}$ 附近只显示(200)峰, 不存在 三方或四方的晶格畸变, 表明样品均为伪立方相结 构 ${ }^{[21-22]}$ 。由于伪立方结构的高对称性, 大大降低了 光在传播过程中由于衍射和双折射所产生的光损耗, 进而提高光学透过率。

图 3 为 $0.825 \mathrm{KNN}-0.175 \mathrm{SLSN}$ 陶瓷样品的表面 扫描电镜照片。从图中可以观察到各个组分 $0.825 \mathrm{KNN}-0.175 \mathrm{SLSN}$ 陶瓷的晶粒结晶性好, 晶粒 堆叠生长, 晶界清晰。当 $x=0.2$ 时, 晶粒间具有较明 显的气孔, 会对样品的致密度产生一定影响, 从而
导致击穿场强降低以及入射光发生散射, 最终影响 样品的储能及透光性能。图 3 的插图为 $0.825 \mathrm{KNN}$ $0.175 \mathrm{SLSN}$ 陶瓷的粒径分布图, 可以发现掺杂 $\mathrm{La}_{2} \mathrm{O}_{3}$ 在一定程度上抑制了 $0.825 \mathrm{KNN}-0.175 \mathrm{SLSN}$ 陶瓷的

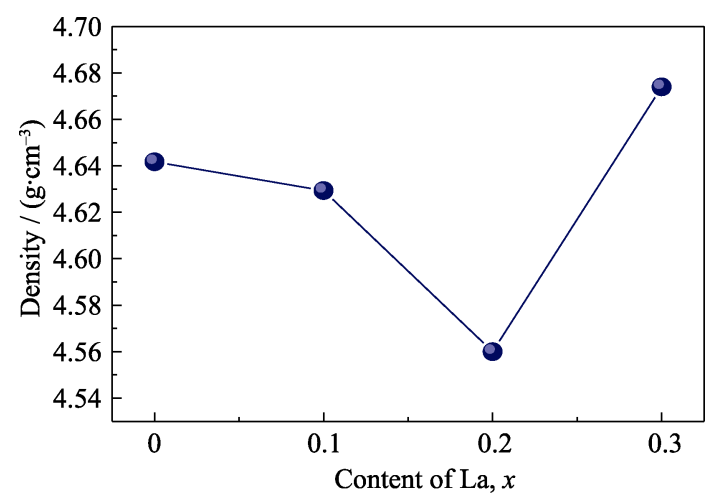

图 $10.825 \mathrm{KNN}-0.175 \mathrm{SLSN}$ 陶瓷密度与 $\mathrm{La}$ 含量的关系图 Fig. 1 Variation of density for the $0.825 \mathrm{KNN}-0.175 \mathrm{SLSN}$ ceramics with different content of $\mathrm{La}$

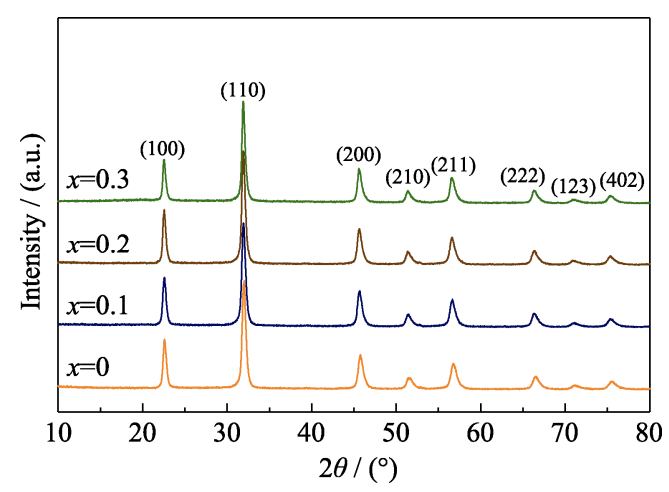

图 $20.825 \mathrm{KNN}-0.175 \mathrm{SLSN}$ 陶瓷在室温下 $2 \theta$ 的 XRD 谱图 Fig. 2 XRD patterns of the $0.825 \mathrm{KNN}-0.175 \mathrm{SLSN}$ ceramics at room temperature
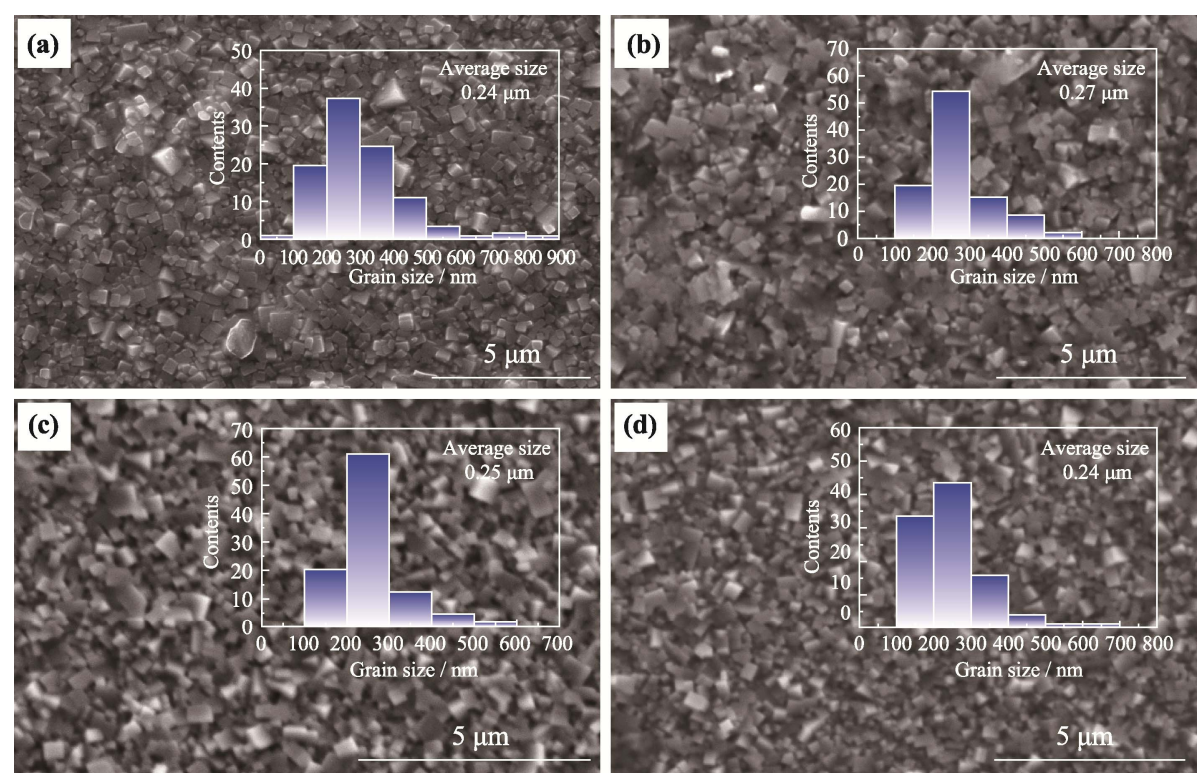

图 $30.825 \mathrm{KNN}-0.175 \mathrm{SLSN}$ 陶瓷室温下自然表面的扫描电镜照片

Fig. 3 SEM micrographs of the original surfaces of $0.825 \mathrm{KNN}-0.175$ SLSN

(a) $x=0$; (b) $x=0.1$; (c) $x=0.2$; (d) $x=0.3$ 
晶粒生长。当 $x=0.3$ 时, 平均晶粒尺寸为 $0.24 \mu \mathrm{m}$ 。 一般来说，透明储能陶瓷的晶粒分布均匀，可降低 入射光的损失并提高样品的击穿场强, 从而提高光 学透过率及储能密度。

\section{$2.20 .825 \mathrm{KNN}-0.175 S L S N$ 陶瓷的光学性能 分析}

在 $0.825 \mathrm{KNN}-0.175 \mathrm{SLSN}$ 体系样品中, $x=0.3$ 的 陶瓷的透明度最高。图 4(a)为 400 1200 nm 波长范 围内 $0.825 \mathrm{KNN}-0.175 \mathrm{SLSN}$ 陶瓷 $(x=0,0.3)$ 的直线透 过率光谱图。图 4(a)的插图为 $0.825 \mathrm{KNN}-0.175 \mathrm{SLSN}$ 陶瓷 $(x=0,0.3)$ 样品的照片。0.825KNN-0.175SLSN 陶瓷经打磨至 $0.3 \mathrm{~mm}$ 并抛光后, $x=0.3$ 陶瓷的光学 透过率在可见光波长 $780 \mathrm{~nm}$ 处为 $65.2 \%$ (较 $x=0$ 时的 $60.2 \%$ 提升了 $8.3 \%$ ), 在近红外波长 $1200 \mathrm{~nm}$ 处的透 过率达 71.5\%。与 $\mathrm{KNN}$ 基储能陶瓷的研究报道 ${ }^{[23-25]}$ 比较, $0.825 \mathrm{KNN}-0.175 \mathrm{SLSN}(x=0.3)$ 陶瓷具有更优 异的透明性，有望取代铅基透明储能材料。

当入射光进入陶瓷材料内部时, 会激发具有一 定能量的电子从价带跃迁到导带, 造成光能量损 失。增大材料的禁带宽度会抑制电子发生跃迁，从 而有利于提高材料的透明度。禁带宽度 $E_{\mathrm{g}}$ 可通过 Tauc 方程得出, 如下式 ${ }^{[26]}$ :

$$
(\alpha h v)^{2}=A\left(h v-E_{\mathrm{g}}\right)
$$

其中, 吸收率 $\alpha$ 和光子频率 $v$ 可以根据以下公式获得:

$$
\begin{gathered}
\alpha=\frac{1}{t} \ln \left(\frac{1}{T}\right) \\
v=\frac{c}{\lambda}
\end{gathered}
$$

其中, $h$ 为普朗克常量 $\left(4.1357 \times 10^{-15} \mathrm{eV}\right), A$ 为常数, $t$ 为 样品厚度, $c$ 为光速 $\left(3 \times 10^{8} \mathrm{~m} / \mathrm{s}\right), T$ 为透过率, $\lambda$ 为波长。

通过对图 4(a)中 $0.825 \mathrm{KNN}-0.175 \mathrm{SLSN}$ 陶瓷样 品的光学透过率曲线进行拟合计算, 可得图 4(b),
由图可知, 当 $x=0.3$ 时 $0.825 \mathrm{KNN}-0.175 \mathrm{SLSN}$ 陶瓷的 禁带宽度 $E_{\mathrm{g}}=2.95 \mathrm{eV}$ 。

\section{$2.30 .825 \mathrm{KNN}-0.175 S L S N$ 陶瓷的介电性能} 分析

图 5(a d ) 为 $0.825 \mathrm{KNN}-0.175 \mathrm{SLSN}$ 陶瓷的介电 性能-温度关系图。随着 $x$ 值增大, 介电常数峰逐渐 从一个转变为两个。当 $x=0.2$ 和 0.3 时, 出现的两个 介电常数峰在 $-75{ }^{\circ} \mathrm{C}$ 和 $100{ }^{\circ} \mathrm{C}$ 附近, 分别对应正交 相向四方相的相变以及四方相向立方相的相变, 这 也印证了 XRD 的测试结果, 室温下 $0.825 \mathrm{KNN}-$ $0.175 \mathrm{SLSN}$ 陶瓷均为伪立方的相结构 ${ }^{[27]} 。 0.825 \mathrm{KNN}$ 0.175 SLSN 陶瓷在室温下的介电损耗低于 0.03 , 有 利于获得优异的储能性能。图 $5(\mathrm{e})$ 为 $0.825 \mathrm{KNN}$ $0.175 \mathrm{SLSN}$ 陶瓷在 $1 \mathrm{kHz}$ 下测试的最大介电常数对 应的温度 $\left(T_{\mathrm{m}}\right)$ 与介电常数值 $\left(\varepsilon_{\mathrm{m}}\right)$ 的关系曲线, 随着 $x$ 增大, $T_{\mathrm{m}}$ 呈现升高的趋势, $\varepsilon_{\mathrm{m}}$ 在 950 1100 之间。

\section{$2.40 .825 \mathrm{KNN}-0.175 \mathrm{SLSN}$ 陶瓷的铁电性能 分析}

图 6 为室温下 $0.825 \mathrm{KNN}-0.175 \mathrm{SLSN}$ 陶瓷样品 在 $150 \mathrm{kV} / \mathrm{cm}, 5 \mathrm{~Hz}$ 下测得的不同组分样品的单极 $P-E$ 曲线, 由图可知, 所有组分的样品都为细电滞 回线, 显现出弛豫铁电体的特征。为了直观观察和 分析 $0.825 \mathrm{KNN}-0.175 \mathrm{SLSN}$ 陶瓷样品极化强度的变 化情况, 根据图 6 中的单极电滞回线进行统计。

图 7 为 $0.825 \mathrm{KNN}-0.175 \mathrm{SLSN}$ 陶瓷的 $P_{\max }$ 和 $P_{\mathrm{r}}$ 与掺杂含量的关系曲线。由图可知, 陶瓷样品的 $P_{\mathrm{r}}$ 均小于 $2 \mu \mathrm{C} / \mathrm{cm}^{2}, P_{\mathrm{r}}$ 越小, 越有利于提高储能效率。 随着 $\mathrm{La}$ 含量增大, $P_{\max }$ 呈增大趋势。当 $x$ 从 0 增至 0.2 时, $0.825 \mathrm{KNN}-0.175 \mathrm{SLSN}$ 陶瓷 $P_{\max }$ 值逐渐从 $10.06 \mu \mathrm{C} / \mathrm{cm}^{2}$ 增大至 $13.12 \mu \mathrm{C} / \mathrm{cm}^{2}$, 可见掺杂适量的 $\mathrm{La}$ 元素可以使 $0.825 \mathrm{KNN}-0.175 \mathrm{SLSN}$ 陶瓷 $P_{\max }$ 提高, 有利于提高材料的储能密度。
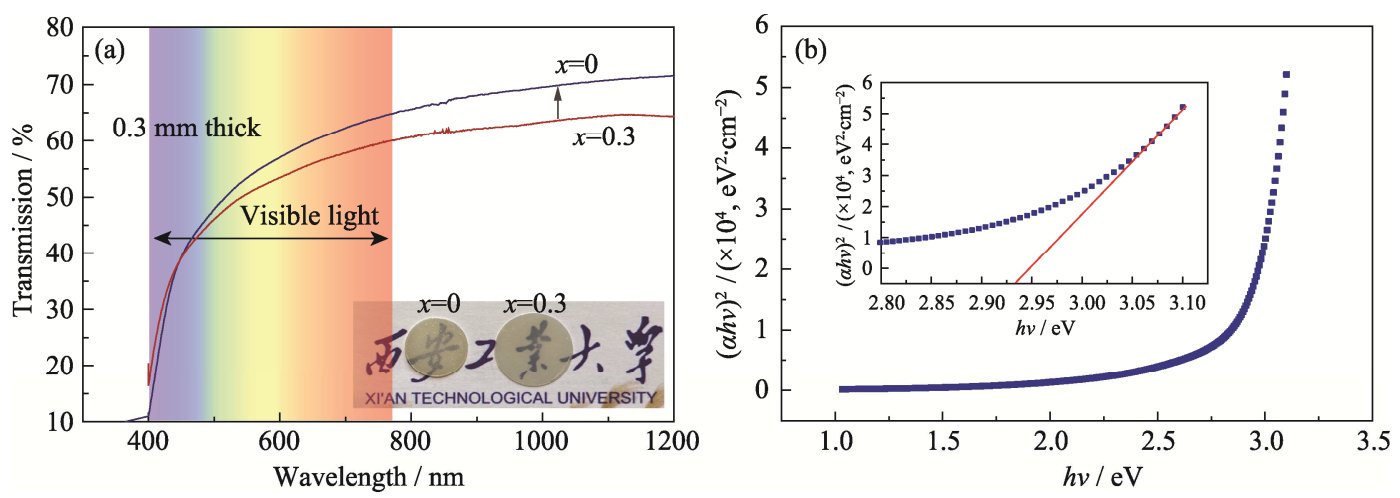

图 $40.825 \mathrm{KNN}-0.175 \mathrm{SLSN}$ 陶瓷的透过率图(a)和 $0.825 \mathrm{KNN}-0.175 \mathrm{SLSN}$ 陶瓷的 $(\alpha h v)^{2}$ 随 $h v$ 的变化关系图(b)

Fig. 4 Transmission spectra (a) of $0.825 \mathrm{KNN}-0.175 \mathrm{SLSN}$ ceramic, with inset showing photographs of the $0.3 \mathrm{~mm}$ specimens, and plots (b) of $(\alpha h v)^{2}$ and $h v$ of the $0.825 \mathrm{KNN}-0.175 \mathrm{SLSN}$ ceramic with $x=0.3$ 

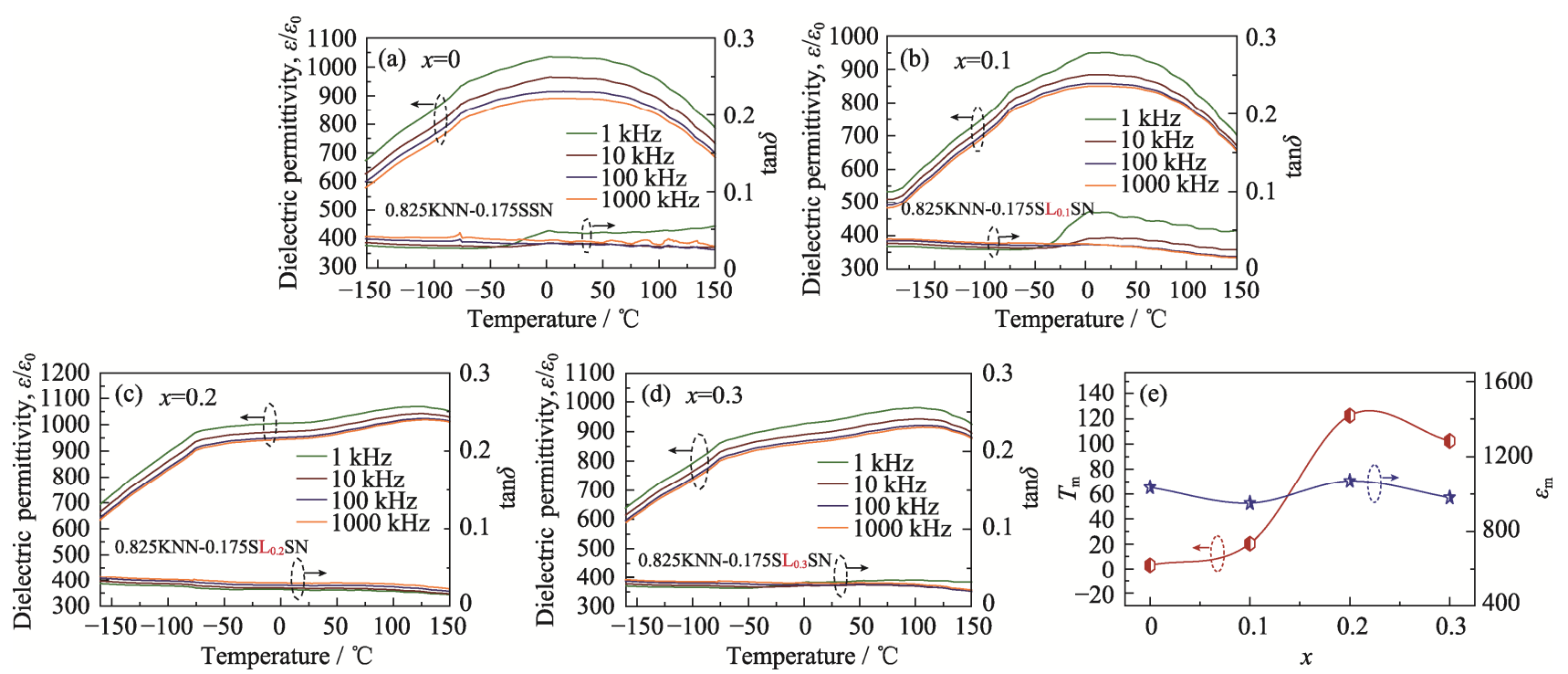

图 $50.825 \mathrm{KNN}-0.175 \mathrm{SLSN}$ 陶瓷样品的介电性能-温度关系图(a d)和 $0.825 \mathrm{KNN}-0.175 \mathrm{SLSN}$ 陶瓷的 $T_{\mathrm{m}}, \varepsilon_{\mathrm{m}}$ 与 $\mathrm{La}$ 掺杂含量关系图(e)

Fig. 5 Temperature dependence of the dielectric properties (a-d), $T_{\mathrm{m}}$ and $\varepsilon_{\mathrm{m}}$ (e) of $0.825 \mathrm{KNN}-0.175 \mathrm{SLSN}$ ceramics

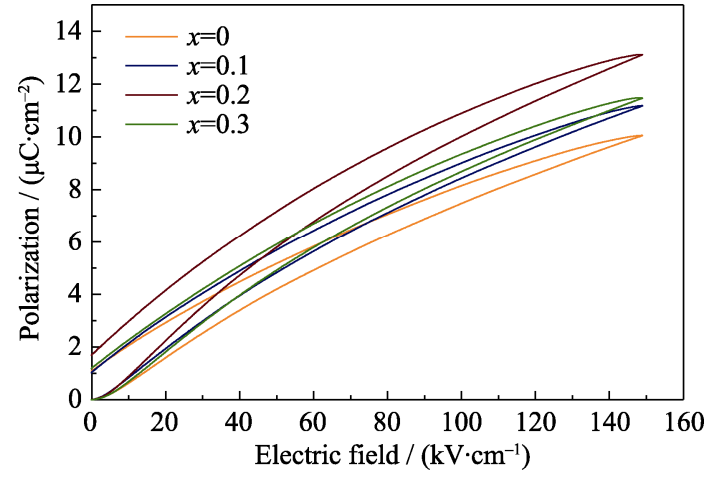

图 $60.825 \mathrm{KNN}-0.175 \mathrm{SLSN}$ 陶瓷在 $150 \mathrm{kV} / \mathrm{cm}$ 电场下的单极 $P-E$ 曲线

Fig. $6 \quad P-E$ loops of $0.825 \mathrm{KNN}-0.175 \mathrm{SLSN}$ ceramics at selected applied electric fields

Colorful figures are available on website

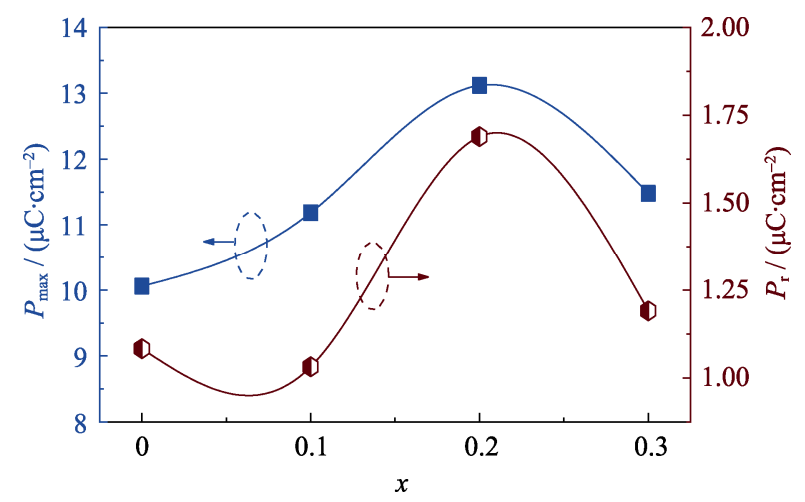

图 7 不同 $\mathrm{La}$ 掺杂含量陶瓷的 $P_{\max }$ 和 $P_{\mathrm{r}}$

Fig. $7 P_{\max }$ and $P_{\mathrm{r}}$ of $0.825 \mathrm{KNN}-0.175 \mathrm{SLSN}$ ceramics as a function of $x$

影响储能密度的另一个重要因素是陶瓷样品的 击穿电场强度 $E_{\mathrm{b}}{ }^{[9,28]} 。 0.825 \mathrm{KNN}-0.175 \mathrm{SLSN}$ 陶瓷在
击穿场强下的单极电滞回线如图 8(a d) 所示。铁电材 料的储能密度和储能效率可通过以下公式获得 ${ }^{[29-31]}$.

$$
\begin{gathered}
W_{\mathrm{rec}}=\int_{P_{\mathrm{r}}}^{P_{\max }} E \mathrm{~d} P \\
W=\int_{0}^{P_{\max }} E \mathrm{~d} P \\
\eta=\frac{W_{\mathrm{rec}}}{W} \times 100 \%
\end{gathered}
$$

其中, $W_{\mathrm{rec}} 、 W 、 P_{\max } 、 P_{\mathrm{r}} 、 E 、 \eta$ 分别表示陶瓷样品 可利用储能密度、储能密度、饱和极化强度、剩余 极化强度、外加电场和储能效率。通过对图 $8(\mathrm{a} \sim \mathrm{d})$ 所得的单极电滞回线进行积分计算, 得到图 8(e)所 示的 $W 、 W_{\mathrm{rec}}$ 随 $x$ 值的变化曲线, 图 8(f) 为 $E_{\mathrm{b}} 、 \eta$ 随 $x$ 值的变化曲线。随着 $\mathrm{La}$ 含量增大, 不同组分陶瓷 的 $W_{\mathrm{rec}}$ 和 $W$ 值呈现逐渐减小之后再增大的趋势。由 于气孔和击穿场强的限制, 在 $x=0.2$ 时储能密度最 低, $W=1.14 \mathrm{~J} / \mathrm{cm}^{3}$ 及 $W_{\mathrm{rec}}=0.95 \mathrm{~J} / \mathrm{cm}^{3}$ 。 $0.825 \mathrm{KNN}$ $0.175 \operatorname{SLSN}(x=0.3)$ 陶瓷具有最优的储能密度 $W=$ $2.25 \mathrm{~J} / \mathrm{cm}^{3}$ 及 $W_{\text {rec }}=1.85 \mathrm{~J} / \mathrm{cm}^{3}$ 。

\section{3 结论}

本研究采用稀土元素 $\mathrm{L} a$ 掺杂改性 $0.825\left(\mathrm{~K}_{0.5} \mathrm{Na}_{0.5}\right) \mathrm{NbO}_{3}-0.175 \mathrm{Sr}\left(\mathrm{Sc}_{0.5} \mathrm{Nb}_{0.5}\right) \mathrm{O}_{3}$ 陶瓷。掺杂 后 $0.825\left(\mathrm{~K}_{0.5} \mathrm{Na}_{0.5}\right) \mathrm{NbO}_{3}-0.175 \mathrm{Sr}_{1-3 x / 2} \mathrm{La}_{x}\left(\mathrm{Sc}_{0.5} \mathrm{Nb}_{0.5}\right) \mathrm{O}_{3}$ $(x=0,0.1,0.2,0.3)$ 陶瓷均具有单一的纯钙钛矿结构, 掺杂 $\mathrm{La}$ 元素并未改变基体材料的相结构, 均为高 对称性的伪立方相, 并在一定程度上抑制晶粒生长, 减小晶粒尺寸。随着 $\mathrm{La}$ 掺杂量增大, $0.825 \mathrm{KNN}$ - 

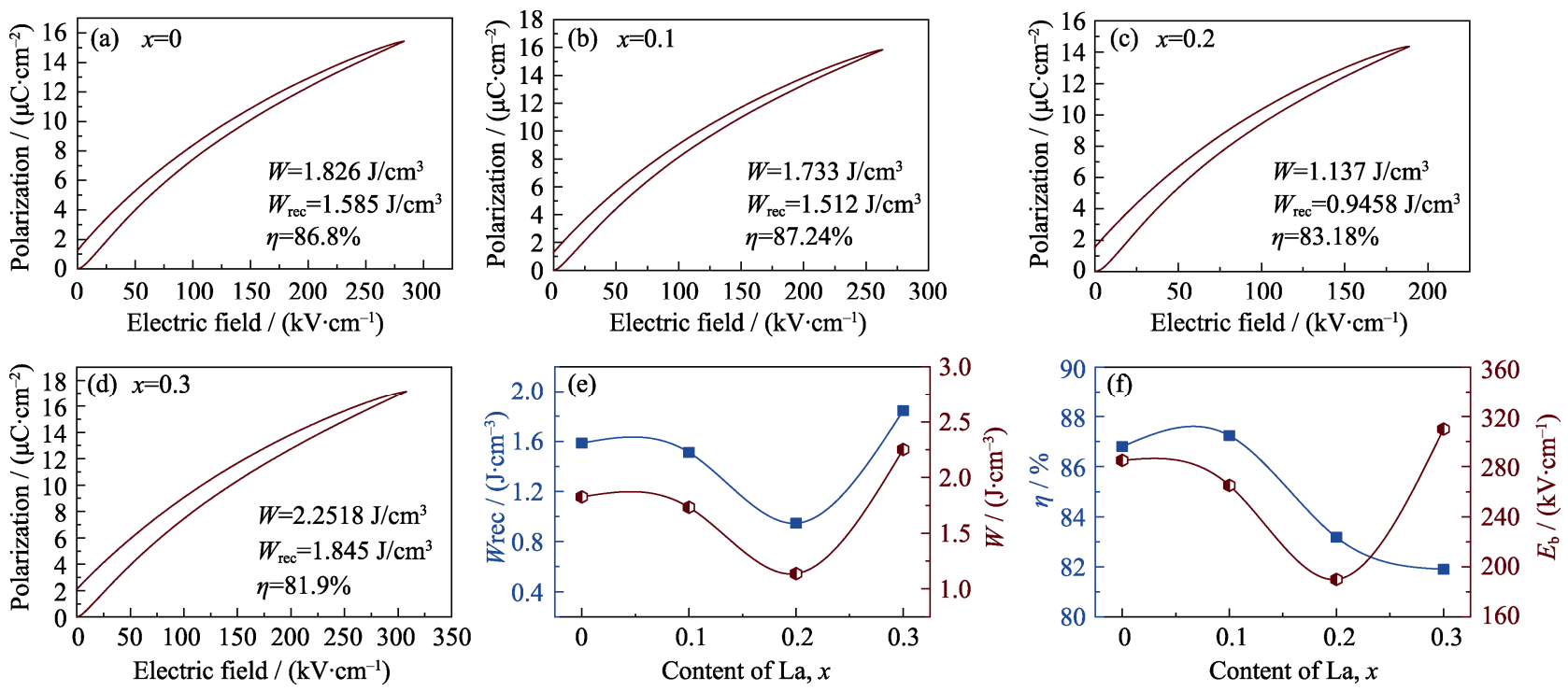

图 $80.825 \mathrm{KNN}-0.175 \mathrm{SLSN}$ 陶瓷在击穿场强下的单极 $P-E$ 曲线 $(\mathrm{a} \sim \mathrm{d}), 0.825 \mathrm{KNN}-0.175 \mathrm{SLSN}$ 陶瓷的 $W 、 W_{\text {rec }}$

与 $x$ 的关系曲线(e), $0.825 \mathrm{KNN}-0.175 \mathrm{SLSN}$ 陶瓷的 $\eta$ 和 $E_{\mathrm{b}}$ 与 $x$ 的关系曲线(f)

Fig. 8 Unipolar $P-E$ hysteresis loops (a-d) of $0.825 \mathrm{KNN}-0.175 \mathrm{SLSN}$ ceramics under different electric fields, variation (e) of $W$ and $W_{\text {rec }}$, for the $0.825 \mathrm{KNN}-0.175 \mathrm{SLSN}$ ceramics with different $x$, and variation

(f) of $\eta$ and $E_{\mathrm{b}}$ for the $0.825 \mathrm{KNN}-0.175 \mathrm{SLSN}$ ceramics with different $x$

\section{$0.175 \mathrm{SLSN}$ 陶瓷饱和极化强度 $P_{\max }$ 呈现增大的趋} 势。在 $x=0.3$ 时, $0.825 \mathrm{KNN}-0.175 \mathrm{SLSN}$ 陶瓷具有优 异的透明性, 在可见光波长 $780 \mathrm{~nm}$ 及近红外波长 $1200 \mathrm{~nm}$ 处透过率分别达 65.2\%及 $71.5 \%$, 同时实现 了最佳的储能特性: $W=2.25 \mathrm{~J} / \mathrm{cm}^{3} 、 W_{\mathrm{rec}}=1.85 \mathrm{~J} / \mathrm{cm}^{3}$ 、 $\eta=81.9 \%$ 。 $0.825 \mathrm{KNN}-0.175 \mathrm{SLSN}$ 陶瓷是有望取代铅 基材料作为透明储能介质材料。

\section{参考文献:}

[1] YAO Z H, SONG Z, HAO H, et al. Homogeneous/inhomogeneousstructured dielectrics and their energy-storage performances. Advanced Materials, 2017, 29(20): 1601727.

[2] YANG L, KONG X, LI F, et al. Perovskite lead-free dielectrics for energy storage applications. Progress in Materials Science, 2019, 102: $72-108$.

[3] WANG H, LIU Y, YANG T, et al. Ultrahigh energy-storage density in antiferroelectric ceramics with field-induced multiphase transitions. Advanced Functional Materials, 2019, 29(7): 1807321.

[4] ZHAO P, WANG H, WU L, et al. High-performance relaxor ferroelectric materials for energy storage applications. Advanced Energy Materials, 2019, 9(17): 1803048.

[5] LI J T, BAI Y, QIN S Q. Direct and indirect characterization of electrocaloric effect in $(\mathrm{Na}, \mathrm{K}) \mathrm{NbO}_{3}$ based lead-free ceramics. Applied Physics Letters, 2016, 109(16): 162902-162904.

[6] WANG X J, WU J G, BRAHIM D. Enhanced electrocaloric effect near polymorphic phase boundary in lead-free potassium sodium niobate ceramics. Applied Physics Letters, 2017, 110(6): 063904.

[7] YANG Z T, GAO F, DU H L, et al. Grain size engineered lead-free ceramics with both large energy storage density and ultrahigh mechanical properties. Nano Energy, 2019, 58: 768-777.

[8] DU H L, YANG Z T, GAO F, et al. Lead-free nonlinear dielectric ceramics for energy storage applications: current status and challenges.
Journal of Inorganic Materials, 2018, 33(10): 1046-1058.

[9] YANG Z T, DU H L, QU S B, et al. Significantly enhanced recoverable energy storage density in potassium-sodium niobatebased lead free ceramics. Journal of Materials Chemistry A, 2016, 4(36): 13778-13785.

[10] SNOW C S. Fabrication of transparent electrooptic PLZT ceramics by atomosphere sintering. Journal of the American Ceramic Society, 1973, 56(2): 91-96.

[11] LI G R, RUAN W, ZENG J T, et al. The effect of domain structures on the transparency of PMN-PT transparent ceramics. Optical Materials, 2013, 35(4): 722-726.

[12] LU X P, XU J W, LING Y, et al. Energy storage properties of $\left(\mathrm{Bi}_{0.5} \mathrm{Na}_{0.5}\right)_{0.93} \mathrm{Ba}_{0.07} \mathrm{TiO}_{3}$ lead-free ceramics modified by $\mathrm{La}$ and $\mathrm{Zr}$ co-doping. Journal of Materiomics, 2016, 2(1): 87-93.

[13] WANG Y F, LV Z L, HUI X, et al. High energy-storage properties of $\left[\left(\mathrm{Bi}_{1 / 2} \mathrm{Na}_{1 / 2}\right)_{0.94} \mathrm{Ba}_{0.06}\right] \mathrm{La}_{(1-x)} \mathrm{Zr}_{x} \mathrm{TiO}_{3}$ lead-free anti-ferroelectric ceramics. Ceramics International, 2014, 40(3): 4323-4326.

[14] REN X, JIN L, PENG Z, et al. Regulation of energy density and efficiency in transparent ceramics by grain refinement. Chemical Engineering Journal, 2020, 390: 124566.

[15] ZHANG M, YANG H, LI D, et al. Excellent energy density and power density achieved in $\mathrm{K}_{0.5} \mathrm{Na}_{0.5} \mathrm{NbO}_{3}$-based ceramics with high optical transparency. Journal of Alloys and Compounds, 2020, 829: 154565.

[16] HEARTLING G S. Improved hot-pressed electrooptic ceramics in the $(\mathrm{Pb}, \mathrm{La})(\mathrm{Zr}, \mathrm{Ti}) \mathrm{O}_{3}$ system. Journal of the American Ceramic Society, 1973, 56(2): 91-96.

[17] SONG Z Z, ZHANG Y C, LU C J, et al. Fabrication and ferroelectric/ dielectric properties of La-doped PMN-PT ceramics with high optical transmittance. Ceramics International, 2017, 43(4): 3720-3725.

[18] ANDREAS K, THOMAS H, JEN K. Transmission physics and consequenees for materials seleetion, manufacturing, and applications. Journal of the European Ceramic Soeiety, 2009, 29(2): 207-221.

[19] PEELEN J, METSELAAR R. Light scattering by pores in polycrystalline materials. Journal of Applied Physics, 1974, 45(1): 216-220.

[20] KRELL A, BLANK P, MA H, et al. Transparent sintered corundum with high hardness and strength. Journal of the American Ceramic 
Society, 2010, 86(1): 12-18.

[21] FU J, ZUO R Z, XU Y D, et al. Investigations of domain switching and lattice strains in $(\mathrm{Na}, \mathrm{K}) \mathrm{NbO}_{3}$-based lead-free ceramics across orthorhombic-tetragonal phase boundary. Journal of the European Ceramic Society, 2017, 37(3): 975-983.

[22] CHENG X J, GOU Q, WU J G, et al. Dielectric, ferroelectric, and piezoelectric properties in potassium sodium niobate ceramics with rhombohedral-orthorhombic and orthorhombic-tetragonal phase boundaries. Ceramics International, 2014, 40(4): 5771-5779.

[23] LIN C, WU X, LIN M, et al. Optical, luminescent and optical temperature sensing properties of $\left(\mathrm{K}_{0.5} \mathrm{Na}_{0.5}\right) \mathrm{NbO}_{3}-\mathrm{ErBiO}_{3}$ transparent ceramics. Journal of Alloys \& Compounds, 2017, 706: 156-163.

[24] GENG Z M, LI K, SHI D L, et al. Effect of Sr and Ba-doping in optical and electrical properties of KNN based transparent ceramics. Journal of Materials Science: Materials in Electronics, 2015, 26(9): 6769-6775.

[25] LIU Z Y, FAN H Q, PENG B L. Enhancement of optical transparency in $\mathrm{Bi}_{2} \mathrm{O}_{3}$-modified $\left(\mathrm{K}_{0.5} \mathrm{Na}_{0.5}\right)_{0.9} \mathrm{Sr}_{0.1} \mathrm{Nb}_{0.9} \mathrm{Ti}_{0.1} \mathrm{O}_{3}$ ceramics for electro-optic applications. Journal of Materials Science, 2015, 50(24): 7958-7966.
[26] WOOTEN F. Optical properties of solids. American Journal of Physics, 1973, 41(7): 939-940.

[27] CHAI Q Z, YANG D, ZHAO X M, et al. Lead-free (K,Na) $\mathrm{NbO}_{3}$ based ceramics with high optical transparency and large energy storage ability. Journal of the American Ceramic Society, 2018, 101(6): 2321-2329.

[28] QU B Y, DU H L, YANG Z T. Lead-free relaxor ferroelectric ceramics with high optical transparency and energy storage ability. Journal of Materials Chemistry C, 2016, 4(9): 1795-1803.

[29] DAI Z H, XIE J L, CHEN Z B, et al. Improved energy storage density and efficiency of $(1-x) \mathrm{Ba}_{0.85} \mathrm{Ca}_{0.15} \mathrm{Zr}_{0.1} \mathrm{Ti}_{0.9} \mathrm{O}_{3}-x \mathrm{BiMg}_{2 / 3} \mathrm{Nb}_{1 / 3} \mathrm{O}_{3}$ lead-free ceramics. Chemical Engineering Journal, 2021, 410: 128341.

[30] DAI Z H, XIE J L, LIU W G, et al. An effective strategy to achieve excellent energy storage properties in lead-free $\mathrm{BaTiO}_{3}$ based bulk ceramics. ACS Applied Materials \& Interfaces, 2020, 12(27): 30289-30296.

[31] DAI Z H, XIE J L, FAN X, et al. Enhanced energy storage properties and stability in $\mathrm{Sr}\left(\mathrm{Sc}_{0.5} \mathrm{Nb}_{0.5}\right) \mathrm{O}_{3}$ modified $0.65 \mathrm{BaTiO}_{3}$ $0.35 \mathrm{Bi}_{0.5} \mathrm{Na}_{0.5} \mathrm{TiO}_{3}$ ceramics. Chemical Engineering Journal, 2020, 397: 125520. 